\title{
Paraplegia as the First Manifestation of Cardiac Tamponade in a Patient after Stent Graft Implantation with Temporary Endocavitary Stimulation
} Artur Milnerowicz, Aleksandra Milnerowicz and Maciej Antkiewicz*

Department and Clinic of Vascular, General and Transplantation Surgery, Jan Mikulicz-Radecki Medical University Hospital, Medical University of Wroclaw, Wroclaw, Poland

\begin{abstract}
The aim of this paper is to present an unusual spectrum of symptoms observed in a patient subjected to implantation of a stent graft to the site of the thoracic-abdominal aortic aneurysm. The patient developed paraplegia which did not result from the spinal cord ischemia but eventually turned out to be a consequence of cardiac tamponade caused by atrial perforation with an endocavitary electrode. A multidisciplinary approach to the hereby reported case prevented severe, potentially fatal complications.
\end{abstract}

Keywords: Paraplegia; Stent graft; Endocavitary electrode; Cardiac tamponade

\section{Introduction}

Cardiovascular diseases constitute one of the most frequent causes of mortality and severe disability in industrialized countries. Availability of aggressive treatments resulted in an increase in survival rates, as well as with a decrease in the percentage of patients who receive a disability pension [1,2]. Angiography became a key diagnostic procedure; its implementation into clinical practice not only has improved the possibilities of causative treatment, but also was reflected by markedly expanded indications for invasive management. As a result, also the patients who were previously disqualified for surgical treatment due to high perioperative risk can be treated nowadays [2].

However, a new group of morbidities emerged, such as postcatheterization aneurysms, obliteration of arteries used for vascular access, hematomas, and other sporadically observed spectacular complications [3].

We report the case of a patient in whom paraplegia was the first manifestation which raised concerns of medical personnel and eventually turned out to be associated with cardiac tamponade. The patient signed a consent form about publication of his data in this article.

\section{Case Report}

A 74-year-old male was admitted to the Clinic of Vascular Surgery for an elective treatment of thoracic-abdominal aortic aneurysm. The primary cause of the pathology was the dissection of the thoracicabdominal aorta associated with a hypertensive crisis which took place in 2013 (Figure 1A). The patient has been implanted a simple stent to the site of the abdominal aortic aneurysm 20 -years earlier. He denied consent for surgical treatment of the aneurysm for a long time, and conservative management resulted in normalization of arterial blood pressure and resolution of pain. A control CT angiography performed in August 2013 (Figure 1B) did not show a clot formation within the false channel; the aneurysm was $43-\mathrm{mm}$ in diameter. The patient still did not give his consent for surgical treatment. Another CT angiography performed in January 2014 (Figure 1C) documented an enlargement of the thoracic aortic aneurysm, up to $52-\mathrm{mm}$ in diameter. The patient still did not give consent for surgical treatment, and the presence of aneurysm was not associated with pain. However, an abdominalthoracic aortic aneurysm, more than $60-\mathrm{mm}$ in diameter, was found during a routine control visit in August 2014 (Figure 1D). In 2015, the patient referred to the Clinic due to non-specific abdominal and thoracic pain. An enlargement of the abdominal aorta at the level of the renal arteries, up to $66-\mathrm{mm}$ in diameter, was documented on a CT angiogram, along with an active false channel at the level of the celiac arteries and diaphragm. Moreover, an enlargement of the thoracic aorta, up to $63-\mathrm{mm}$ in diameter, was observed (Figure 1E). Furthermore, an enlargement of the abdominal aortic aneurysm was observed at the site of the previously implanted simple stent. The patient eventually consented for surgery and was qualified to a multi-stage endovascular treatment: implantation of a stent graft to the thoracic aorta, followed by the treatment of the area of the celiac artery origin.

Due to the evidence of arrhythmia prior to the stent graft implantation to the thoracic aorta, a temporary endocavitary stimulation was implemented. Both procedures were uneventful. The endocavitary electrodes were removed one day after the surgery. Two hours after removal of the electrodes, a decrease in arterial blood pressure was documented, from $130 / 70-\mathrm{mmHg}$ to $100 / 70-\mathrm{mmHg}$, along with a decrease in systolic/diastolic amplitude.

Four hours after the electrode removal, the patient reported an inability to move the feet, along with lower limb dysesthesia. An anti-edematous treatment was started and a CT angiography was performed. The latter revealed accumulation of fluid in the pericardial cavity (Figure 2). Due to further exacerbation of the ailments and gradual deterioration of vital parameters, namely a decrease in arterial blood pressure and systolic/diastolic amplitude and acceleration of heart rate, an immediate pericardiocentesis was performed. A total of $200-\mathrm{mL}$ of bloody fluid was evacuated from the pericardial cavity

*Corresponding author: Maciej Antkiewicz, MD, Department of Vascular General and Transplantation Surgery, University Hospital in Wroclaw, Borowska 213, Wroclaw, Poland, Tel: 0048 664142018; Fax: 004871733 2009; E-mail: maciej.antkiewicz@gmail.com

Received July 15, 2016; Accepted July 27, 2016; Published August 03, 2016

Citation: Milnerowicz A, Milnerowicz A, Antkiewicz M (2016) Paraplegia as the First Manifestation of Cardiac Tamponade in a Patient after Stent Graft Implantation with Temporary Endocavitary Stimulation. J Vasc Med Surg 4: 278. doi: 10.4172/23296925.1000278

Copyright: (c) 2016 Milnerowicz A, et al. This is an open-access article distributed under the terms of the Creative Commons Attribution License, which permits unrestricted use, distribution, and reproduction in any medium, provided the original author and source are credited. 

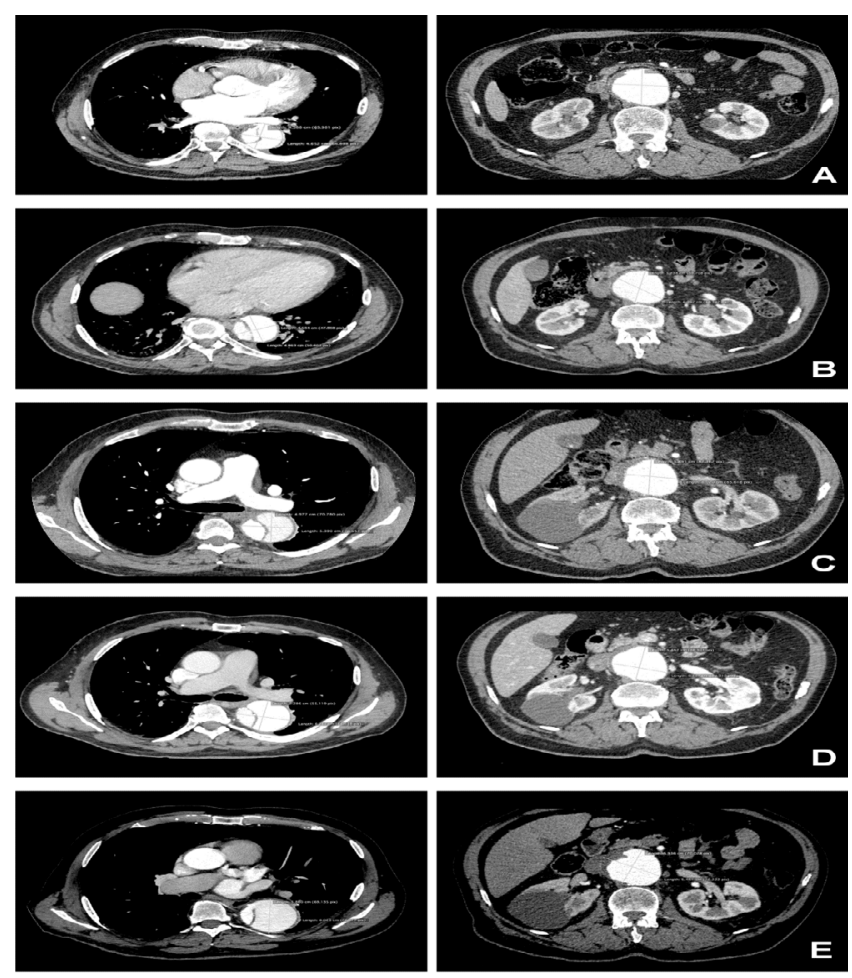

Figure 1: Computed tomography scans show progression of the thoracicabdominal aortic aneurysm diameter. A: in May 2013, diameter $(\mathrm{mm})$ of the thoracic and abdominal aorta respectively: $49 \times 47$ and $55 \times 55$. B: August 2013, $50 \times 47$ and $57 \times 60$. C: January $2014,50 \times 54$ and $58 \times 60$. D: August 2014, $53 \times 62$ and $56 \times 62$. E: in January 2015, $59 \times 60$ and $59 \times 64$.

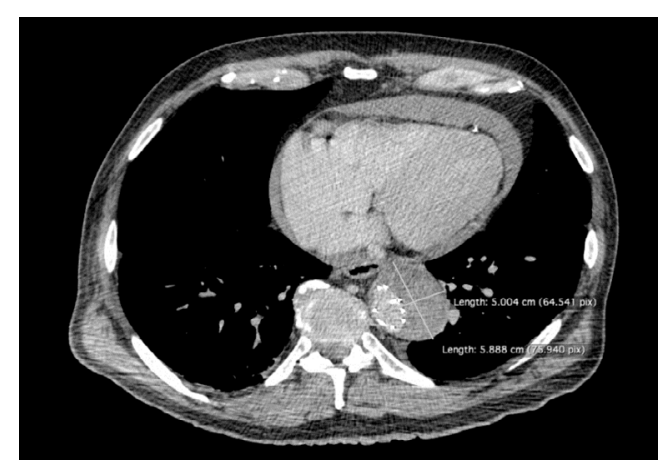

Figure 2: Computed tomography scan show accumulation of fluid in the pericardial cavity.

during the procedure. A $6 \mathrm{~F}$ sheath was left in the pericardial cavity in an event of blood re-accumulation.

A return of the lower limb function was observed immediately after the pericardiocentesis, along with a normalization of vital parameters. Ten hours following the puncture, the patient presented with full motor capacity of lower extremities.

A total of $100-\mathrm{mL}$ and $30-\mathrm{mL}$ of bloody fluid were evacuated from the pericardial cavity on the second and third postoperative day, respectively, before the sheath was removed. The patient was discharged home in a good status on the $6^{\text {th }}$ postoperative day.

In November 2015, the patient was re-admitted to the Clinic for the second stage of the treatment, i.e. implantation of a custom-made stent graft (Jotec, Warsaw, Poland) to the thoracic-abdominal aorta. There was no need to insert an endocavitary electrode as the patient's cardiologic status was good. The second stage of the treatment was uneventful.

\section{Discussion}

Individuals with thoracic-abdominal aortic dissecting aneurysm constitute a specific group of patients [1,2]. The risk of postoperative paraplegia cannot be assessed unequivocally due to the considerable variability in the origin of the artery of Adamkiewicz and heterogeneity in the spinal cord blood supply. Previous implantation of a simple stent to the site of the abdominal aortic aneurysm additionally compromised arterial supply of the spinal cord. Therefore, the presence of paraplegia on postoperative day 2 might raise a suspicion of the spinal cord ischemia resulting from occlusion of the spinal cord arteries [2].

The diagnosis was eventually established on the basis of a spiral CT contrast angiography. Cardiac tamponade was not considered as a primary cause of the paraplegia, and neither the decrease in arterial blood pressure nor the increases in heart rate were spectacular enough to raise a suspicion of this pathology, especially that the latter progressed slowly before eventually manifesting as a paraplegia.

\section{Conclusion}

The hereby presented case report illustrates that paraplegia observed after stent graft implantation is not necessarily an irreversible complication resulting from occlusion of the spinal cord supplying arteries by the stent.

\section{Conflict of Interest}

None: This research did not receive any specific grant from funding agencies in the public, commercial, or not-for-profit sectors.

\section{References}

1. Taylor PR, Gaines PA, McGuinness CL, Cleveland TJ, Beard JD, et al. (2001) Thoracic Aortic Stent Grafts - Early Experience from Two Centres using Commercially Available Devices. Eur J Vasc Endovasc Surg 22: 70-76.

2. Hughes GC, Barfield ME, Shah AA, Williams JB, Kuchibhatla M, et al. (2012) Staged total abdominal debranching and thoracic endovascular aortic repair for thoracoabdominal aneurysm. J Vasc Surg 56: 621-629.

3. Etz DC, Luehr M, Aspern KV, Misfeld M, Gudehus S, et al. (2004) Spinal cord ischemia in open and endovascular thoracoabdominal aortic aneurysm repair: new concepts. J Cardiovasc Surg (Torino) 55: 159-168. 